Volume 129, Number 2, Pages 389-395

S 0002-9939(00)05596-9

Article electronically published on August 29, 2000

\title{
INVARIANT LINEAR MANIFOLDS FOR CSL-ALGEBRAS AND NEST ALGEBRAS
}

\author{
ALAN HOPENWASSER
}

(Communicated by David R. Larson)

\begin{abstract}
Every invariant linear manifold for a CSL-algebra, $\operatorname{Alg} \mathcal{L}$, is a closed subspace if, and only if, each non-zero projection in $\mathcal{L}$ is generated by finitely many atoms associated with the projection lattice. When $\mathcal{L}$ is a nest, this condition is equivalent to the condition that every non-zero projection in $\mathcal{L}$ has an immediate predecessor $\left(\mathcal{L}^{\perp}\right.$ is well ordered). The invariant linear manifolds of a nest algebra are totally ordered by inclusion if, and only if, every non-zero projection in the nest has an immediate predecessor.
\end{abstract}

Kadison's transitivity theorem [6] implies that a $\mathrm{C}^{*}$-algebra acting on a Hilbert space which has no non-trivial closed invariant subspaces must also have no nontrivial invariant linear manifolds. This note investigates the analogous situation for CSL-algebras and, in particular, for nest algebras. We identify exactly the family of CSL-algebras for which every invariant linear manifold is, in fact, a closed subspace (and hence an element of the lattice of invariant subspaces for the algebra). When specialized to nest algebras, this family reduces to those nest algebras for which each non-zero element of the nest has an immediate predecessor. Note that a nest, $\mathcal{L}$, satisfies this condition if, and only if, the nest, $\mathcal{L}^{\perp}$, consisting of orthogonal complements of elements of $\mathcal{L}$ is well ordered. For any other nest algebra, not only are there invariant linear manifolds which are not closed, but the family of invariant linear manifolds is not totally ordered by inclusion. Thus, most nest algebras are "nest algebras" in a topological sense but not an algebraic sense.

Some of the results in this paper were obtained by Foias in 1971/72 in his study of invariant operator ranges 3 , 4 or by Ong in his description of all the invariant operator ranges for a nest algebra [9]. Davidson extended this description to CSLalgebras in [1]. Davidson's book on nest algebras [2] is a convenient reference for most of this work on operator ranges. All the same, we give complete and independent proofs for our results on arbitrary linear manifolds which follow from prior work on operator ranges; these proofs are shorter and more elementary than the arguments via operator ranges. (The price to be paid is that these results are somewhat weaker than the operator range results. The converses, on the other hand, are correspondingly stronger.)

Received by the editors June 15, 1998 and, in revised form, April 8, 1999.

2000 Mathematics Subject Classification. Primary 47L35.

Key words and phrases. Nest algebra, CSL-algebra, invariant subspace, invariant linear manifold.

The author would like to thank Ken Davidson for drawing his attention to the references regarding operator ranges. 
To fix notation, let $\mathcal{L}$ be a commutative lattice of orthogonal projections acting on a Hilbert space $\mathcal{H}$. It is assumed that $\mathcal{L}$ is complete and that it contains 0 and $I$. The algebra of all bounded linear operators on $\mathcal{H}$ which leave invariant each projection in $\mathcal{L}$ is denoted by $\operatorname{Alg} \mathcal{L}$. It is convenient to use the same symbol to denote both a subspace and the orthogonal projection whose range is the subspace. The term "subspace" will always mean "closed linear subspace"; the term "linear manifold" is used when closure is not assumed. Thus $\mathcal{L}$ will be viewed simultaneously as a complete lattice of closed subspaces of $\mathcal{H}$ and as a lattice of mutually commuting projections in $\mathcal{B}(\mathcal{H})$ which is closed in the strong operator topology (and which contains 0 and $I$ ).

The primary tool used in this note is the necessary and sufficient condition on two vectors $x$ and $y$ in $\mathcal{H}$ for the existence of an operator $T$ in $\operatorname{Alg} \mathcal{L}$ such that $T x=y$ : with the understanding that the fraction $0 / 0$ is to be interpreted as 0 , the existence of an operator $T$ carrying $x$ to $y$ is equivalent to

$$
\sup _{E \in \mathcal{L}} \frac{\left\|E^{\perp} y\right\|}{\left\|E^{\perp} x\right\|}<\infty .
$$

This was first proved by Lance [7] for nest algebras and then extended to CSLalgebras in [5].

Recall that an atom, $A$, associated with a subspace lattice, $\mathcal{L}$, is a minimal nonzero interval from $\mathcal{L}$; i.e., $A$ has the form $P-Q$ where $P, Q \in \mathcal{L}, Q<P$, and $A F$ is either 0 or $A$, for all $F \in \mathcal{L}$. If $A_{1}, A_{2}, \ldots$ is a sequence of atoms from $\mathcal{L}$ (either a finite or an infinite sequence) let $E\left(A_{1}, A_{2}, \ldots\right)$ denote the smallest projection in $\mathcal{L}$ which contains each of the atoms $A_{1}, A_{2}, \ldots$ In other words,

$$
E\left(A_{1}, A_{2}, \ldots\right)=\bigwedge_{F \in \mathcal{L}}\left\{F \mid A_{n} \subseteq F, \text { for all } n\right\} .
$$

In the following definition, and throughout this paper, when we say that a projection in $\mathcal{L}$ is generated by a set of atoms, we mean that it is the smallest projection in $\mathcal{L}$ which contains each of the atoms of the set.

Definition. A commutative subspace lattice, $\mathcal{L}$, is hyperatomic if every non-zero projection in $\mathcal{L}$ is generated by finitely many atoms.

Remark. If $P=E\left(A_{1}, \ldots, A_{n}\right)$, then we may as well assume that the atoms $A_{1}, \ldots, A_{n}$ are independent in the sense that $A_{i} E\left(A_{j}\right)=0$ whenever $i \neq j$. (Just delete some atoms from the list, if necessary.)

If $P$ is a non-zero projection in $\mathcal{L}$, the projection $\bigvee\{F \in \mathcal{L} \mid F \nsupseteq \nexists P\}$ will be denoted by $P_{-}$. If $x$ and $y$ are vectors in $\mathcal{H}$, then there is a rank-one operator $T \in A \lg \mathcal{L}$ such that $T x=y$ if, and only if, $x \in P_{-}^{\perp}$ and $y \in P$ for some $P \in \mathcal{L}$. For nests, this was proven by Ringrose [10]; the extension to commutative lattices is due to Longstaff [8]. Note that if $A$ is an atom from $L$ and if $P=E(A)$, then $A \leq P_{-}^{\perp}$. Consequently, if $x \in A$ and $y \in P$, then there is a rank-one operator in $\operatorname{Alg} \mathcal{L}$ such that $T x=y$.

In the theorem which follows, operator range refers to the range of a bounded linear operator acting on $\mathcal{H}$. 
Theorem. Let $\mathcal{L}$ be a commutative subspace lattice acting on a separable Hilbert space. The following conditions are equivalent:

(1) $\mathcal{L}$ is hyperatomic.

(2) Every ascending sequence, $F_{1} \leq F_{2} \leq \ldots$, of projections in $\mathcal{L}$ is eventually constant.

(3) Every invariant operator range for $\operatorname{Alg} \mathcal{L}$ is a closed subspace (and therefore an element of $\mathcal{L})$.

(4) Every invariant linear manifold for $\operatorname{Alg} \mathcal{L}$ is a closed subspace (and therefore an element of $\mathcal{L})$.

If $\mathcal{L}$ is a nest, then the following additional conditions are equivalent to each of the conditions above:

(5) Every non-zero projection in $\mathcal{L}$ has an immediate predecessor.

(6) The invariant operator ranges for $\operatorname{Alg} \mathcal{L}$ are totally ordered by inclusion.

(7) The invariant linear manifolds for $\operatorname{Alg} \mathcal{L}$ are totally ordered by inclusion.

Proof. (11) $\Rightarrow(2)$ : Let $F_{1} \leq F_{2} \leq \ldots$ be an ascending sequence of projections in $\mathcal{L}$. Let $P=\bigvee F_{j}$. Since $\mathcal{L}$ is hyperatomic, there are finitely many atoms, $A_{1}, \ldots, A_{n}$, so that $P=E\left(A_{1}, \ldots, A_{n}\right)$. Each of these atoms must be a subprojection of some $F_{j}$; since there are only finitely many atoms in this list, there is $j$ such that $A_{i} \leq F_{j}$, for all $i=1, \ldots, n$. But then $P=F_{j}$; hence $P=F_{k}$, for all $k \geq j$.

(2) $\Rightarrow(11)$ : Assume that $\mathcal{L}$ is not hyperatomic. Let $P \in \mathcal{L}$ be a projection which is not generated by finitely many atoms. Let $Q$ be the smallest projection in $\mathcal{L}$ which contains all atoms which are subprojections of $P$. First, suppose that $Q<P$. If $F$ is any element of $\mathcal{L}$ such that $Q \leq F<P$, then there is a projection $G \in \mathcal{L}$ such that $F<G<P$ (since $P-F$ is not an atom from $\mathcal{L}$ ). A routine induction argument now yields an ascending sequence of projections which is not eventually constant. Next, suppose that $Q=P$. In other words, there is a sequence $A_{1}, A_{2}, \ldots$ (necessarily infinite) of atoms such that $P=E\left(A_{1}, A_{2}, \ldots\right)$. For each $n$, let $F_{n}=E\left(A_{1}, \ldots, A_{n}\right)$. Then $F_{1}, F_{2}, \ldots$ is an ascending sequence of projections in $\mathcal{L}, F_{n}<P$ for all $n$, and $\bigvee F_{n}=P$. Thus, this sequence is not eventually constant.

(4) $\Rightarrow(3)$ is immediate and (3) $\Rightarrow(2)$ follows from [2, Theorem 15.29]. However, we give an elementary proof of (4) $\Rightarrow(2)$ which does not require any information about operator ranges. Suppose (2) is false. Let $0<F_{1}<F_{2}<\ldots$ be a strictly increasing sequence of projections in $\mathcal{L}$. For each $n$, let $x_{n} \in F_{n}-F_{n-1}$ be a nonzero vector chosen so that $\sum_{n=1}^{\infty} n^{2}\left\|x_{n}\right\|^{2}<\infty$. (For example, let $x_{n}$ be any vector in $F_{n}-F_{n-1}$ for which $\left\|x_{n}\right\|=1 / n^{2}$.)

For each $n$, let $y_{n}=n x_{n}$. By the choice of the $x_{n}$, the sequence, $y_{n}$, is a square summable sequence of mutually orthogonal vectors. Let $x=\sum_{n=1}^{\infty} x_{n}$ and $y=\sum_{n=1}^{\infty} y_{n}$. (Both sums converge in $\mathcal{H}$.) Let $\mathcal{M}_{x}=\{T x \mid T \in \operatorname{Alg} \mathcal{L}\}$, an invariant linear manifold for $\operatorname{Alg} \mathcal{L}$. For each $n$,

$$
\begin{aligned}
& F_{n}^{\perp} x=\sum_{k=n+1}^{\infty} x_{k} \text { and } \\
& F_{n}^{\perp} y=\sum_{k=n+1}^{\infty} y_{k}=\sum_{k=n+1}^{\infty} k x_{k} .
\end{aligned}
$$


So,

$$
\begin{aligned}
\left\|F_{n}^{\perp} y\right\|^{2} & =\sum_{k=n+1}^{\infty} k^{2}\left\|x_{k}\right\|^{2} \geq \sum_{k=n+1}^{\infty}(n+1)^{2}\left\|x_{k}\right\|^{2} \\
& =(n+1)^{2} \sum_{k=n+1}^{\infty}\left\|x_{k}\right\|^{2}=(n+1)^{2}\left\|F_{n}^{\perp} x\right\|^{2} .
\end{aligned}
$$

Thus,

$$
\frac{\left\|F_{n}^{\perp} y\right\|}{\left\|F_{n}^{\perp} x\right\|} \geq n+1
$$

and hence,

$$
\sup _{F \in \mathcal{L}} \frac{\left\|F^{\perp} y\right\|}{\left\|F^{\perp} x\right\|}=\infty
$$

This shows that $y \notin \mathcal{M}_{x}$.

On the other hand, $\left(F_{n}-F_{n-1}\right) x=x_{n} \in \mathcal{M}_{x}$, for all $n$, whence $y_{n}=n x_{n} \in \mathcal{M}_{x}$ and $\sum_{n=1}^{K} y_{n} \in \mathcal{M}_{x}$, for all $K$. Since $y=\lim _{K \rightarrow \infty} \sum_{n=1}^{K} y_{n}$, this shows that $y \in \overline{\mathcal{M}_{x}}$. Thus, $\mathcal{M}_{x}$ is an invariant linear manifold which is not closed.

(1) $\Rightarrow$ (4): First, we show that if $x \in \mathcal{H}$, then $\mathcal{M}_{x}$ is closed; i.e., every singly generated invariant linear manifold is closed. Let $x \in \mathcal{H}$ and $P=\overline{\mathcal{M}_{x}}$. If $x=0$, then $P=0$; so assume $x \neq 0$ and, hence, $P \neq 0$. Since $\mathcal{L}$ is hyperatomic, there exist atoms $A_{1}, \ldots, A_{n}$ so that $P=E\left(A_{1}, \ldots, A_{n}\right)$. Without loss of generality, we may assume that $A_{1}, \ldots, A_{n}$ are independent; i.e., that $A_{i} E\left(A_{j}\right)=0$ whenever $i \neq j$. Equivalently, $A_{i} T A_{j}=0$ for all $T \in \operatorname{Alg} \mathcal{L}$, when $i \neq j$. It follows that $A_{j} x \neq 0$, for all $j$.

If $y \in E\left(A_{j}\right)$, then, by the comments preceding the statement of the theorem, there is $T \in \operatorname{Alg} \mathcal{L}$ such that $y=T A_{j} x$. Thus $E\left(A_{j}\right) \subseteq \mathcal{M}_{x}$, for all $j$.

If $y \in P$ is arbitrary, then, since $P=E\left(A_{1}\right) \vee \cdots \vee E\left(A_{n}\right)$, there exist $y_{i} \in E\left(A_{i}\right)$ such that $y=y_{1}+\cdots+y_{n}$. Since each $y_{i} \in \mathcal{M}_{x}$, we have $y \in \mathcal{M}_{x}$. This shows that $P=\overline{\mathcal{M}_{x}} \subseteq \mathcal{M}_{x}$, so $\mathcal{M}_{x}$ is closed.

Before turning to general invariant linear manifolds, we need an observation: if $P_{1}=\mathcal{M}_{x_{1}}$ and $P_{2}=\mathcal{M}_{x_{2}}$, then there is a vector $x$ such that $\mathcal{M}_{x}=P_{1} \vee P_{2}$. Indeed, choose $x=x_{1}+P_{1}^{\perp} x_{2}$. Since $x_{1}=P_{1} x$, we have $P_{1} \subseteq \mathcal{M}_{x}$. Let $y \in P_{2} P_{1}^{\perp}$. Since $P_{2}=\mathcal{M}_{x_{2}}$, there is $T \in \operatorname{Alg} \mathcal{L}$ such that $y=T x_{2}$. Since $x_{2}=P_{1}^{\perp} x_{2}+P_{1} x_{2}$,

$$
y=T x_{2}=T P_{1}^{\perp} x_{2}+T P_{1} x_{2}=T P_{1}^{\perp} x_{2}+P_{1} T P_{1} x_{2} .
$$

However, $P_{1}^{\perp} y=y$, so

$$
y=P_{1}^{\perp} T P_{1}^{\perp} x_{2}=P_{1}^{\perp} T P_{1}^{\perp}\left(x_{1}+P_{1}^{\perp} x_{2}\right)=P_{1}^{\perp} T P_{1}^{\perp} x .
$$

Thus, $P_{2} P_{1}^{\perp} \subseteq \mathcal{M}_{x}$. Since $P_{1} \vee P_{2}=P_{1}+P_{2} P_{1}^{\perp}$, we have $P_{1} \vee P_{2} \subseteq \mathcal{M}_{x} \subseteq P_{1} \vee P_{2}$; i.e., $\mathcal{M}_{x}=P_{1} \vee P_{2}$.

Finally, let $\mathcal{M}$ be an arbitrary invariant linear manifold for $\operatorname{Alg} \mathcal{L}$. Let $P=\overline{\mathcal{M}}$; so $P=\bigvee\left\{\mathcal{M}_{x} \mid x \in \mathcal{M}\right\}$. Since $\mathcal{H}$ is separable, we can write $P$ as the join of countably many subspaces of the form $\mathcal{M}_{x}$; i.e., there is a sequence, $x_{1}, x_{2}, \ldots$, of vectors in $\mathcal{M}$ so that $P=\bigvee_{j=1}^{\infty} P_{j}$, where $P_{j}=\mathcal{M}_{x_{j}}$, all $j$. The observation above shows that $P_{1} \vee P_{2}$ and, indeed, any finite join $P_{1} \vee \cdots \vee P_{n}$ can be written in the form $\mathcal{M}_{x}$ for some $x \in \mathcal{M}$. So we may assume that $P_{1} \leq P_{2} \leq \ldots$ Since $\mathcal{L}$ is hyperfinite and we have already shown that $(\mathbb{1}) \Rightarrow(2)$, this sequence is eventually 
constant. But this shows that $P=\mathcal{M}_{x_{j}}$, for some $x_{j} \in \mathcal{M}$. Thus $P=\mathcal{M}$ and $\mathcal{M}$ is closed.

For the rest of the proof we assume that $\mathcal{L}$ is a nest.

The equivalence, (1) $\Leftrightarrow(5)$, is trivial; in a nest a projection is generated by finitely many atoms if, and only if, it is generated by a single atom. (1) $\Rightarrow(7)$ follows immediately from (1) $\Rightarrow(4)$ and $(7) \Rightarrow(6)$ is trivial.

(6) $\Rightarrow(21)$ : Assume that (2) is false; let $0=F_{0}<F_{1}<F_{2}<\ldots$ be a strictly increasing sequence of projections in $\mathcal{L}$. Suppose that $\lambda=\left(\lambda_{n}\right)$ is a decreasing sequence of positive real numbers. Let $D_{\lambda}=\sum_{n=1}^{\infty} \lambda_{n}\left(F_{n}-F_{n-1}\right)$ (the sum converges in the strong operator topology). By a result of Ong [9], the range of $D_{\lambda}$ is an invariant operator range for $\operatorname{Alg} \mathcal{L}$.

If $x$ is a vector in $\bigvee_{n=1}^{\infty} F_{n}$, let $x_{n}=\left(F_{n}-F_{n-1}\right) x$, for each $n$. Necessarily, $\sum_{n=1}^{\infty}\left\|x_{n}\right\|^{2}<\infty$. Note that $x$ is in the range of $D_{\lambda}$ if, and only if, $\sum_{n=1}^{\infty} \frac{1}{\lambda_{n}{ }^{2}}\left\|x_{n}\right\|^{2}$ $<\infty$.

In order to exhibit two operator ranges which are not related by inclusion, choose two decreasing seqences, $\lambda$ and $\mu$, of positive real numbers in such a way that $\mu_{n} / \lambda_{n} \geq n$ whenever $n$ is even and $\lambda_{n} / \mu_{n} \geq n$ whenever $n$ is odd. Next, choose vectors $x_{n}$ and $y_{n}$ in $F_{n}-F_{n-1}$ so that $\left\|x_{n}\right\|=\mu_{n} / n$ and $\left\|y_{n}\right\|=\lambda_{n} / n$, for all $n$. The four sequences, $\left(x_{n}\right),\left(\frac{1}{\mu_{n}} x_{n}\right),\left(y_{n}\right)$, and $\left(\frac{1}{\lambda_{n}} y_{n}\right)$ are all square summable. Consequently, $x=\sum_{n=1}^{\infty} x_{n}$ and $y=\sum_{n=1}^{\infty} y_{n}$ are convergent sums; $x$ is an element of the range of $D_{\mu}$; and $y$ is an element of the range of $D_{\lambda}$.

Now consider the sequences $\left(\frac{1}{\lambda_{n}} x_{n}\right)$ and $\left(\frac{1}{\mu_{n}} y_{n}\right)$. For $n$ even,

$$
\left\|\frac{x_{n}}{\lambda_{n}}\right\|=\left(\frac{\mu_{n}}{\lambda_{n}}\right)\left(\frac{1}{\mu_{n}}\right)\left\|x_{n}\right\| \geq 1
$$

while, for $n$ odd,

$$
\left\|\frac{y_{n}}{\mu_{n}}\right\|=\left(\frac{\lambda_{n}}{\mu_{n}}\right)\left(\frac{1}{\lambda_{n}}\right)\left\|y_{n}\right\| \geq 1
$$

This shows that $x$ is not in the range of $D_{\lambda}$ and $y$ is not in the range of $D_{\mu}$; thus $D_{\lambda}$ and $D_{\mu}$ are not ordered by inclusion.

$(7) \Rightarrow(2)$ : This is evident from the preceeding argument and the trivial implication (7) $\Rightarrow(6)$. However, here is an alternative proof which avoids the use of operator ranges and which exhibits two singly generated invariant linear manifolds which are unrelated by inclusion.

Let $e_{n}=1+2+\cdots+n$, for all $n$. Since $e_{n}=e_{n-1}+n$, we have, for all $k$,

$$
\begin{aligned}
\sum_{n=k+1}^{\infty} \frac{1}{2^{e_{n}}} & <\sum_{n=0}^{\infty} \frac{1}{2^{e_{k+1}+n}}=\sum_{n=0}^{\infty} \frac{1}{2^{e_{k}+k+1+n}} \\
& =\frac{1}{2^{e_{k}}} \sum_{n=0}^{\infty} \frac{1}{2^{k+1+n}}=\frac{1}{2^{e_{k}} 2^{k}}
\end{aligned}
$$


Define two sequences:

$$
\begin{aligned}
& a_{n}= \begin{cases}0, & \text { if } n \text { is even; } \\
1 / 2^{e_{n}}, & \text { if } n \text { is odd; }\end{cases} \\
& b_{n}= \begin{cases}1 / 2^{e_{n}}, & \text { if } n \text { is even; } \\
0, & \text { if } n \text { is odd }\end{cases}
\end{aligned}
$$

In other words,

$$
\begin{aligned}
a & =\left(\frac{1}{2^{e_{1}}}, 0, \frac{1}{2^{e_{3}}}, 0, \frac{1}{2^{e_{5}}}, 0, \ldots\right), \\
b & =\left(0, \frac{1}{2^{e_{2}}}, 0, \frac{1}{2^{e_{4}}}, 0, \frac{1}{2^{e_{6}}}, \ldots\right) .
\end{aligned}
$$

Now assume that $k$ is an odd integer. Then

$$
\sum_{n=k}^{\infty} b_{n}=\sum_{n=k+1}^{\infty} b_{n}<\sum_{n=k+1}^{\infty} \frac{1}{2^{e_{n}}}<\frac{1}{2^{e_{k}} 2^{k}} .
$$

Therefore,

$$
\left(\sum_{n=k}^{\infty} b_{n}\right)^{-1}>2^{k} 2^{e_{k}}
$$

Since we also have

$$
\sum_{n=k}^{\infty} a_{n}>\frac{1}{2^{e_{k}}}
$$

we obtain

$$
\left(\sum_{n=k}^{\infty} a_{n}\right)\left(\sum_{n=k}^{\infty} b_{n}\right)^{-1}>2^{k}, \quad \text { for all odd } k
$$

Similarly,

$$
\left(\sum_{n=k}^{\infty} b_{n}\right)\left(\sum_{n=k}^{\infty} a_{n}\right)^{-1}>2^{k}, \quad \text { for all even } k
$$

Thus we have

$$
\begin{aligned}
& \sup _{k}\left(\sum_{n=k}^{\infty} a_{n}\right)\left(\sum_{n=k}^{\infty} b_{n}\right)^{-1}=\infty, \quad \text { and } \\
& \sup _{k}\left(\sum_{n=k}^{\infty} b_{n}\right)\left(\sum_{n=k}^{\infty} a_{n}\right)^{-1}=\infty .
\end{aligned}
$$

Assume that $\mathcal{L}$ fails to satisfy (2) and let $0=F_{0}<F_{1}<F_{2}<\ldots$ be a strictly increasing sequence of projections in $\operatorname{Alg} \mathcal{L}$. For each $n \geq 1$, choose vectors $x_{n}$ and $y_{n}$ in $F_{n}-F_{n-1}$ so that $\left\|x_{n}\right\|^{2}=a_{n}$ and $\left\|y_{n}\right\|^{2}=b_{n}$; finally, let $x=\sum_{n=1}^{\infty} x_{n}$ and $y=\sum_{n=1}^{\infty} y_{n}$. Since $\left\|F_{k}^{\perp} x\right\|^{2}=\sum_{n=k+1}^{\infty} a_{n}$ and $\left\|F_{k}^{\perp} y\right\|^{2}=\sum_{n=k+1}^{\infty} b_{n}$, it follows that

$$
\sup _{k} \frac{\left\|F_{k}^{\perp} x\right\|}{\left\|F_{k}^{\perp} y\right\|}=\infty \quad \text { and } \quad \sup _{k} \frac{\left\|F_{k}^{\perp} y\right\|}{\left\|F_{k}^{\perp} x\right\|}=\infty .
$$


Thus

$$
\sup _{F \in \mathcal{L}} \frac{\left\|F^{\perp} x\right\|}{\left\|F^{\perp} y\right\|}=\infty \quad \text { and } \quad \sup _{F \in \mathcal{L}} \frac{\left\|F^{\perp} y\right\|}{\left\|F^{\perp} x\right\|}=\infty .
$$

This shows that for all $T \in \operatorname{Alg} \mathcal{L}, T y \neq x$ and $T x \neq y$. The linear manifolds $\mathcal{M}_{x}$ and $\mathcal{M}_{y}$ are invariant under $\operatorname{Alg} \mathcal{L}$ and, since $x \notin \mathcal{M}_{y}$ and $y \notin \mathcal{M}_{x}$, we have $\mathcal{M}_{x} \nsubseteq \mathcal{M}_{y}$ and $\mathcal{M}_{y} \nsubseteq \mathcal{M}_{x}$.

\section{REFERENCES}

[1] K. R. Davidson, Invariant operator ranges for reflexive algebras, J. Operator Theory 7 (1982), 101-107. MR 83e:47004

[2] _ N N Nest algebras, Pitman Research Notes in Mathematics Series, vol. 191, Longman Scientific and Technical, 1988. MR 90f: 47062

[3] C. Foiaş, Invariant para-closed subspaces, Indiana Univ. Math. J. 20 (1971), 897-900. MR 53:3734

[4] _ Invariant para-closed subspaces, Indiana Univ. Math. J. 21 (1972), 887-906. MR 45:2516

[5] A. Hopenwasser, The equation $T x=y$ in a reflexive operator algebra, Indiana Univ. Math. J. 29 (1980), 121-126. MR 81c:47014

[6] R. V. Kadison, Irreducible operator algebras, Proc. Nat. Acad. Sci. U.S.A. 43 (1957), 273-276. MR 19:47e

[7] E. C. Lance, Some properties of nest algebras, Proc. London Math. Soc. (3) 19 (1969), 45-68. MR 39:3325

[8] W. Longstaff, Strongly reflexive lattices, J. London Math. Soc. (2) 11 (1975), 491-498. MR 52:15036

[9] S.-C. Ong, Invariant operator ranges of nest algebras, J. Operator Theory 3 (1980), 195-201. MR 81f: 47008

[10] J. Ringrose, On some algebras of operators, Proc. London Math. Soc. (3) 15 (1965), 61-83. MR 30:1405

Department of Mathematics, University of Alabama, Tuscaloosa, Alabama 35487

E-mail address: ahopenwa@euler.math.ua.edu 